\title{
Brunet, M. et Romero-Torres, A. (2021). La gestion de projets au Québec. Des cas pour illustrer une expertise en croissance. Éditions JFD.
}

\section{Christophe Bredillet ${ }^{\mathrm{a}}$}

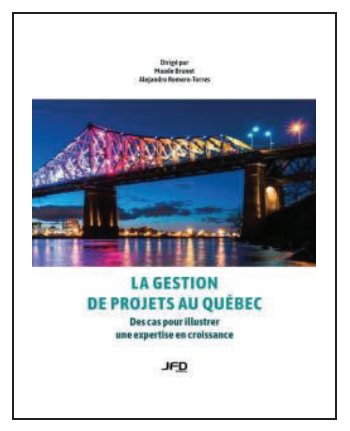

Comme le titre l'indique, ce livre s'inscrit dans la lignée des ouvrages intégrant un ancrage empirique à partir de cas remarquables et l'apprentissage réflexif mobilisant des fondements théoriques solides. Les thèmes retenus, qui forment les cinq sections de l'ouvrage, font l'objet d'une attention toujours soutenue, car ils correspondent à des enjeux importants en pratique.

L'ouvrage est organisé en une introduction, suivie de cinq sections comprenant 17 chapitres.

Dans cette recension, nous nous devons d'insister sur la riche introduction de Brunet (HEC Montréal) et Romero-Torres (ESG UQAM), qui nous rappellent d'abord l'importance des projets dans le développement de la société, à savoir la "projectification» de la société. Les auteurs notent qu'au Québec, le territoire couvert par l'ouvrage, 13 milliards de dollars seront investis annuellement en infrastructures publiques au cours des 10 prochaines années, ce qui nécessitera le développement de compétences et de pratiques adaptées aux enjeux de création de valeur économique, environnementale et sociale.

Devant ces enjeux, Brunet et Romero-Torres soulignent l'expertise historique québécoise en gestion de projets et l'écosystème unique sur le plan international, qui comprend associations professionnelles, universités et environnement socio-politico-économique. À titre d'exemple, ils citent le développement des programmes aux cycles supérieurs en gestion de projets dans le réseau de l'Université du Québec, il y a environ 45 ans. C'était alors une première mondiale. Ce développement a par ailleurs donné naissance à un réseau de recherche extrêmement fertile, dont l'influence a été et est internationale, par exemple les travaux de Miller et Lessard ou encore d'Aubry et Hobbs.

Pour conclure, les auteurs définissent le cadre intégrateur de la gestion de projets utilisé pour structurer l'ouvrage, cadre inspiré de Cha et ses collègues (2018). Ce cadre articule les cinq catégories de pratiques correspondant aux cinq sections de l'ouvrage, soit 1) définition de projet, 2) gouvernance de projet (incluant les relations contractuelles), 3) livraison de projet, 4) transfert des résultats du projet et 5) compétences humaines.

La section 1, qui s’intéresse à la «définition de projet», est illustrée par trois cas, chacun faisant l'objet d'un chapitre. Deux pratiques clés sont mises en évidence. La première est l'importance de la bonne compréhension des enjeux politiques par les gestionnaires dans l'analyse des projets d'infrastructure publique en s'appuyant sur des approches de l'analyse des politiques publiques diverses (rationnelles, pluralistes, cognitives et critiques) [voir chapitre 1 - Le développement des grands projets publics et les théories des politiques publiques : le cas du Réseau structurant de transport en commun de Québec par Pierre-André Hudon (Université Laval)].

a Professeur titulaire en gestion organisationnelle de projet, Université du Québec à Trois-Rivières

Revue Organisations \& Territoires • Volume $30 \cdot \mathrm{N}^{\circ} \mathbf{2} \cdot 2021$

181 
La seconde pratique clé est l'utilisation d'approches participatives, tant sur le plan de la gouvernance du processus de consultation publique que dans l'établissement des besoins préalable à la définition d'un projet [voir chapitre 2 - Le processus de consultation publique du REM : un projet à la gouvernance inédite par Maude Brunet (HEC Montréal), Nathalie Drouin (ESG UQAM) et Philippe Gauthier (HEC Montréal); et chapitre 3 - L'implication des parties prenantes dans une approche participative Lean: le projet du nouveau complexe hospitalier de Québec par Hafsa Chbaly (ÉTS), Maude Brunet (HEC Montréal), Éric Daneau (CHU de Québec) et Daniel Forgues (ÉTS)].

La «gouvernance de projet» est l'objet de la section 2. Trois cas sont proposés. Ils permettent de faire ressortir deux pratiques importantes. La première est la nécessité d'une compréhension intime des interactions entre acteurs (parties prenantes et intervenants) et de leur bon équilibre. Cette pratique est mise en évidence par l'analyse, d'une part, de la dynamique de mise en place de conditions favorisant l'acceptabilité sociale [voir chapitre 4 - Développement de partenariats avec les Premières Nations dans un grand projet hydroélectrique : le cas du projet Eastmain-1-A-Sarcelle-Rupert à la Baie-James par Sofiane Baba (Université de Sherbrooke), Réal Courcelles (Hydro-Québec) et Marc Dunn (Niskamoon)] et, d'autre part, de l'influence des acteurs en position d'autorité dans le succès d'un modèle d'implantation d'un projet [voir chapitre 5 - Le cas de l'implantation du système de gestion intégrée de la performance dans les organisations de santé au Québec par Jean-Sébastien Marchand et Mylaine Breton (Université de Sherbrooke)].

La seconde pratique importante se concentre sur la mise en place de mécanismes favorisant une relation contractuelle collaborative [voir chapitre 6-L'influence de la modélisation des données sur la gestion des parties prenantes: le cas du projet d'agrandissement de l'aéroport de Québec par Tais Scherer (ÉTS) et Maude Brunet (HEC Montréal)].

La section 3 nous conduit à explorer les pratiques de «livraison de projet». Les cinq cas présentés permettent de mettre en exergue trois pratiques principales. La première concerne les approches ou démarches mises en œuvre pour une meilleure création de valeur, et qui combinent les aspects « classiques » et « agiles », le tout dans une perspective d'effectuation [voir chapitre 7 - La mise en projet d'une initiative d'innovation : le cas de la télédermatologie pour le dépistage des cancers de la peau par Marie-Pierre Faure et Nathalie Ross (Institut TransMedTech) et Mélissa Trépanier, Patrick Cohendet, Philip Mathieu et Laurent Simon (HEC Montréal); chapitre 8 - Gestion de projet agile hors TI : le cas de l'onganisation d'un événement par Julie Delisle (ESG UQAM); et chapitre 11 - La revitalisation de la Tour de Montréal et les pratiques innovantes en construction par Joëlle Brodeur (Parc olympique) et Maude Brunet (HEC Montréal)].

La deuxième pratique souligne l'importance des arrangements contractuels adaptés entre acteurs [voir chapitre 9 - Les modes de réalisation comme moyen d'orchestrer les projets de construction: concepts et exemples nationaux par Mario Bourgault (Polytechnique Montréal), Ivanka Iordanova (ÉTS) et Nathalie Perrier, Sara Rankohi et Mathieu Denis (Polytechnique Montréal)].

Enfin, la troisième pratique exige une connaissance approfondie des ressorts sous-jacents des parties prenantes, à savoir de leurs intérêts et référents. Elle apparait comme une condition clé à une bonne gestion de ces dernières [voir chapitre 10 - La réalisation du projet d'illumination du pont Jacques-Cartier et la complexité de la gestion des parties prenantes par Maude Brunet et Philippe Gauthier (HEC Montréal)].

Le « transfert des résultats du projet» est étudié en section 4. Les trois cas discutés font ressortir trois pratiques ou principes clés. Premièrement, on constate un principe d'équifinalité au regard de la performance parmi des projets similaires faisant l'objet d'un design organisationnel propre, mais adapté à leur contexte [voir chapitre 12 - Le design organisationnel, la performance et l'équifinalité : une étude des trois projets majeurs de transformation des CHU à Montréal par Monique Aubry (ESG UQAM), Marie-Claire Richer et Mélanie Lavoie-Tremblay (Université McGill), Claude Fortin et Jean-François Fortin Verreault].

Deuxièmement, nonobstant le choix de l'approche, l'organisation de la gestion de la réalisation des bénéfices obéit à quelques principes fondamentaux [voir chapitre 13 - L'art de gérer la réalisation des bénéfices d'un projet: l'expérience de la Société de transport de Montréal par Magalie Paré et Alejandro Romero-Torres (ESG UQAM)]. 
Troisièmement, la prise en compte d'approches plurielles, dont la géopolitique, enrichit la réflexion autour des projets [voir chapitre 14 - Enrichir la recherche et la formation en gestion de projet par l'entremise de l'approche géopolitique? Réflexion à partir du cas des gaz de schiste au Québec par Nicolas Paquet et Mario Carrier (Université Laval)].

La section 5 couvre un ensemble de pratiques transversales par rapport aux précédentes : les « compétences humaines». Les deux pratiques essentielles soulignées par les trois cas présentés ne paraittront pas nécessairement nouvelles au lecteur, ce qui n'enlève rien à leur importance.

D’abord, la capacité à faire collaborer des parties prenantes et des acteurs divers (nous ne dirons pas « gérer les parties prenantes », expression trop restrictive et normative à notre goût), qui met l'humain au cœur des processus d'émergence et de changements induits par les projets, est une pratique fondamentale [voir chapitre 15 - Compétences et stratégies de gestion pour les projets publics gérés en partenariat : le cas du projet les Jardins Gamelin du Partenariat du Quartier des spectacles par Marie-Pierre Leroux et Caroline Coulombe (ESG UQAM) et Jozef FleuryBerthiaume (Partenariat du Quartier des spectacles); et chapitre 16 - Le projet de refonte du programme de formation en gestion de projet chez.Hydro-Québec Innovation, équipement et services partagés : quelles leçons pour la gestion du changement d'un projet de développement organisationnel par Caroline Coulombe et Marie-Pierre Leroux (ESG UQAM), Émilie Sénéchal (Hydro-Québec), Véronique Duguay et Daphnée Daniel].

La seconde pratique fondamentale soulignée est la capacité à adapter les approches de gestion de projet à la diversité et à la pluralité des contextes et situations de chaque projet. Ainsi, une solution unique ne convient pas à tous [voir chapitre 17 - Gestion de projet événementiel sous la lentille du "Making Project Critical": le projet des Grands Feux du Casino du Lac-Leamy par Julie Bérubé et Audrey-Ann Tully de Cotret (UQO)].

Cet ouvrage, d'une lecture aisée, combine harmonieusement les dimensions didactique, narrative et réflective, ce qui siéra à différents lecteurs. Une de ses forces est la diversité du choix des cas, que ce soit les secteurs couverts, les types de projets, leur état d'avancement ou encore leur niveau de performance. Une autre force vient du fait que les auteurs viennent à la fois de la pratique et de la recherche, ce qui rend les pratiques mises en exergue d'autant plus fondées dans une perspective d'études pragmatiques.

En conclusion, les directeurs de l'ouvrage Maude Brunet et Alejandro Romero-Torres ainsi que les différents collaborateurs nous proposent un ouvrage de grande qualité apportant un regard riche sur les enjeux associés à la "projectification» croissante de la société. Il est utile autant à la réflexion qu’à l'action. Cette contribution de haute volée sera profitable à un large lectorat, fût-il praticien, chercheur, étudiant ou personne engagée dans le débat politique.

Pour consulter le site de l'éditeur concernant cet ouvrage : www.editionsjfd.com/gdp 